\title{
Hopping Trajectory Planning for Asteroid Surface Exploration Accounting for Terrain Roughness*
}

\author{
Ziwen LI, ${ }^{1)}$ Xiangyuan ZenG, ${ }^{1 \dagger}$ and Shuquan $\mathrm{WANG}^{2)}$ \\ ${ }^{1)}$ School of Automation, Beijing Institute of Technology, Beijing 100081, China \\ ${ }^{2)}$ Key Laboratory of Space Utilization, Chinese Academy of Sciences, Beijing 100094, China
}

\begin{abstract}
Hopping rovers have become a promising way of asteroid surface exploration. This paper focuses on the hopping trajectory design between two given surface points and discusses the irregular terrain's influence on the design process. By taking the hopping rover as a point mass, dynamical equations are derived based on the polyhedral method. The principle of hopping trajectory planning is summarized with the related solving algorithm. The initial velocity increments required to control the subsequent hopping trajectories are determined based on parabolic motion. The numerical simulations apply a triaxial ellipsoid to approximate comet 133P/Elst-Pizarro preliminarily. The smooth and rocky polyhedron models of the ellipsoid are constructed, respectively. With the two models, the different initial conditions' hopping trajectories are planned and compared to verify the proposed planning method and discuss the influence of terrain roughness on the trajectory design.
\end{abstract}

Key Words: Hopping Trajectory Planning, Space Exploration, Asteroid Surface Exploration, Rocky Polyhedron Model

\section{Nomenclature}

$\boldsymbol{E}_{e}$ : edge dyad

$\boldsymbol{F}_{f}:$ facet dyad

$\boldsymbol{g}$ : gravitational acceleration

$G$ : universal gravitational constant

$L_{e}$ : line integral associated with the edge

$[L]$ : distance unit for normalization

$\boldsymbol{n}$ : normal direction outside the plane

$N$ : magnitude of the normal force

$p$ : hopping number

$r$ : position

$\boldsymbol{r}_{e}$ : vector from $\boldsymbol{r}$ to any point on the edge

$\boldsymbol{r}_{f}$ : vector from $\boldsymbol{r}$ to any point on the facet

$t_{f}$ : hopping transfer time

$t$ : tangential direction

$T_{\mathrm{A}}$ : rotation period of the asteroid

$[T]$ : time unit for normalization

$U$ : gravitational potential

$V$ : effective potential

$V$ : velocity

$\Delta \boldsymbol{V}$ : velocity increment

$\Delta V$ : magnitude of the velocity increment

$s$ : position error

$s_{\mathrm{h}}$ : threshold of the position error

$\alpha$ : angular acceleration of the asteroid

$\varepsilon$ : restitution coefficient

$\theta_{f}$ : solid angle corresponding to the facet

$\kappa$ : dynamical parameter of the asteroid

(C) 2021 The Japan Society for Aeronautical and Space Sciences *Received 25 September 2020; final revision received 14 December 2020; accepted for publication 21 December 2020.

†Corresponding author, zeng@bit.edu.cn $\mu_{\mathrm{k}}$ : coefficient of the kinetic friction

$\sigma$ : density of the asteroid

$\omega$ : rotation angular velocity of the asteroid

Subscripts

0 : initial

$E S$ : set of all edges of the asteroid

$F S$ : set of all triangular facets of the asteroid

in: ingoing

nin: normal component of the ingoing vector

out: outgoing

$t$ : tangential direction

tin: tangential component of the ingoing vector

$Z$ : component of the $Z$ coordinate

$\because$ relative derivative to time

: unit vector

': relative derivative to the normalized time

: normalized vector

\section{Introduction}

Surface exploration is an efficient method to obtain small celestial bodies' characteristics and structures, providing more accurate data for the scientific analysis of planetary evolution. ${ }^{1,2)}$ Generally, a hopping mechanism is adopted for traveling across the asteroid surface in low gravity. Compared with the rover propelled by normal wheels or crawlers, the hopping rover has better kinematic dexterity and will not float upwards as soon as it begins to move. ${ }^{3)}$ Additionally, changes in the velocity of the hopping rover can reveal asteroid surface mechanical properties, such as the compressive strength, material distribution characteristics, thermal inertia, and so on. ${ }^{4)}$

Some asteroid missions have released a hopping rover or 
lander to explore the surface. The European Space Agency (ESA) launched the Rosetta spacecraft in March 2004. ${ }^{5)}$ Philae, the lander of Rosetta, successfully researched the topography and material composition of comet $67 \mathrm{P}$, although it experienced three unexcepted bounces and became trapped in a crack before losing contact with Rosetta. ${ }^{6)}$ To explore the surface of the asteroid 162173 Ryugu, the MINERVAII consisting of three rovers (i.e., rover-1A, rover-1B, and MINERVA-II-2) were attached to the spacecraft Hayabusa2. ${ }^{7,8)}$ MINERVA-II-1 containing rover-1A and rover$1 \mathrm{~B}$, were the first successful probes to travel over the surface of Ryugu using a hopping mechanism. However, rover MINERVA-II-2 failed to work before deployment, and was instead dropped to analyze the microgravity field of Ryugu. ${ }^{9,10)}$

Aiming for future surface explorations, hopping trajectory planning that considers complex terrains for the surface probes should be a critical issue if a hopping rover is required/expected to arrive at a predetermined location. Bellerose and Scheeres ${ }^{11)}$ utilized the control method for an unmanned aerial vehicle (UAV) to counteract the effect of surface equilibria. Liu et al. ${ }^{12)}$ studied hopping trajectory optimization from the perspective of energy and proposed a guidance algorithm based on convex optimization. Hockman and Pavone, ${ }^{13)}$ developed an algorithm searching for singlehopping trajectories over the surface of asteroid 25143 Itokawa, which was solved applying the Lambert problem. Similarly, based on the Lambert problem, Kalita and Thangavelautham ${ }^{14)}$ obtained a multiple-hopping path utilizing several single-hopping arcs with the maximum hopping constraint. The above studies have analyzed the trajectories of rovers from different perspectives. However, these works all neglected the influence of irregular asteroid terrain on the trajectory. The high-resolution images from the surface surveys illustrate complex asteroid terrains, such as cobbles, gravel, rocks, regolith, and impact craters. ${ }^{15-17)}$ Because the hopping rover is usually small, perhaps only a few tens of centimeters, such as the MINERVA-II, ${ }^{18)}$ it is insufficient to plan a hopping trajectory over an ideally smooth surface without considering rough terrain.

This paper proposes a new method for hopping trajectory planning near asteroid surfaces. The rocky polyhedron model is generated and applied to simulate the irregular asteroid topography primitively. Given a terrain environment with the initial surface point $\mathrm{A}$ and the target surface point B, the hopping rover is treated as a point mass located at point A at the beginning time. The trajectory design method's practical objective is to search for the appropriate velocity increments that allow the particle to hop from the initial point A to the destination point $\mathrm{B}$.

The rest of this paper is organized as follows. Section 2.1 presents the dynamical equations near the asteroid surface and the numerical simulation of the collision. Section 2.2 introduces the hopping design principle and the corresponding algorithm. Section 2.3 illustrates the generation process of the rocky polyhedron model. Section 3 plans the hopping trajectories over the smooth and rocky ellipsoid model surfaces, re- spectively. Finally, conclusions are summarized in Section 4.

\section{Model and Concept}

This section presents the dynamical equations and the collision model. The principle and the core algorithm of the hopping trajectory design are summarized. Afterward, rocky polyhedron model generation is illustrated. In this study, asteroid microgravity, centrifugal force, normal surface force, and friction are analyzed. In contrast, solar radiation pressure (SRP) and third-body perturbing forces are neglected.

\subsection{Dynamical models}

Dynamical equations are the foundation of particle motion, constructed by the orbital segment(s) and surface segment(s). The driven events, such as lift-off and landing, trigger the transfer between the two motion modes. ${ }^{19)}$ For the convenience of analyzing trajectory evolution, the hopping rover's shape and structure are ignored, and the rover is simplified as a particle preliminarily. However, the simplification may lose some accuracy when describing the trajectory evolution without considering the change in attitude. With the realistic structure's influence, the hopping rover may decelerate to zero quickly when it switches to surface motion after colliding with the asteroid surface. For example, MINERVA-II had temperature sensors on the edges that look like thorns. ${ }^{20)}$ When the rover was about to land, these projections and the scattered rocks became significant obstacles to its surface motion. Therefore, the surface segment is neglected in this study temporarily, with the hopping rover simplified as a particle to analyze its locomotion.

Figure 1 illustrates the event-driven model. With a large enough velocity increment, the surface hopping rover will lift off and switch to orbital motion. ${ }^{21)}$ Afterward, several collisions between the rover and the asteroid surface will happen unless the initial velocity increment exceeds the asteroid's escape velocity directly. During this process, if the rover bounces after the collision, its motion still belongs to the orbital motion (or ballistic motion). If the rover lands after the collision, the orbital motion is terminated.

During orbital motion, assuming that a particle is only attracted by the gravity of the central body, referred to as nat-

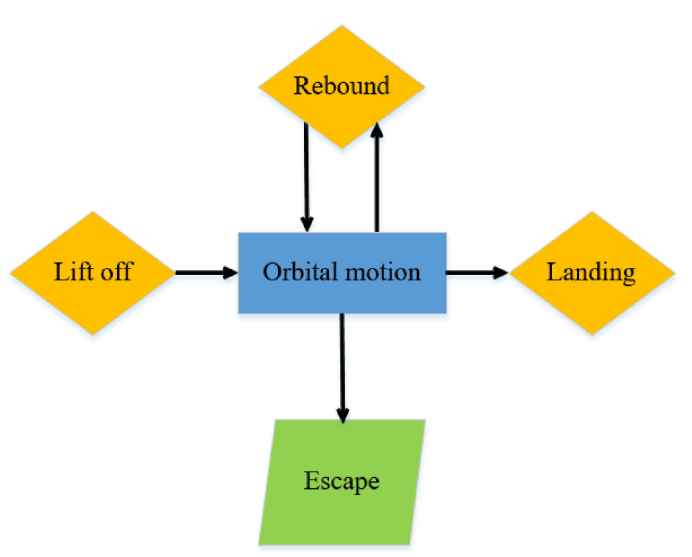

Fig. 1. Event-driven model of the near-surface motion. 
ural motion, its dynamical equations can be expressed as: ${ }^{22)}$

$$
\left\{\begin{array}{l}
\ddot{\boldsymbol{r}}+2 \boldsymbol{\omega} \times \dot{\boldsymbol{r}}+\boldsymbol{\omega} \times(\boldsymbol{\omega} \times \boldsymbol{r})+\boldsymbol{\alpha} \times \boldsymbol{r}=-\nabla U \\
-\nabla U=G \sigma\left(\sum_{e \in E S} L_{e} \boldsymbol{E}_{e} \cdot \boldsymbol{r}_{e}-\sum_{f \in F S} \theta_{f} \boldsymbol{F}_{f} \cdot \boldsymbol{r}_{f}\right)
\end{array}\right.
$$

where $\boldsymbol{r}$ is the position vector from the mass center of a small body to the particle, $-\nabla U$ is the gravitational acceleration, $\omega$ is the rotation angular velocity of the central body, and $\alpha$ is its corresponding angular acceleration. Since most small bodies rotate along a fixed axis, $\boldsymbol{\alpha}$ usually approximates to zero. The explicit expression of $-\nabla U$ is given by adopting the polyhedral method. ${ }^{23)}$ Here, the subscript ES represents the set of all edges, $F S$ represents the set of all triangular sides of the small body, $\boldsymbol{r}_{e}$ is the vector from the position $\boldsymbol{r}$ to any point on the edge $e$, and $\boldsymbol{r}_{f}$ is the vector from $\boldsymbol{r}$ to any point on the side $f$. The scalar parameter $L_{e}$ is the line integral associated with the edge $e$ of side $f, \theta_{f}$ is the solid angle corresponding to side $f, \sigma$ is the small body's density, and $G$ is the gravitational constant.

The concept of effective potential $V$ is applied to simplify Eq. (1):

$$
V=-(\boldsymbol{\omega} \times \boldsymbol{r}) \cdot(\boldsymbol{\omega} \times \boldsymbol{r}) / 2+U
$$

Substituting Eq. (2) into Eq. (1), the most commonly used expression of orbital motion yields

$$
\ddot{r}+2 \omega \times \dot{r}=-\nabla V
$$

In the calculation, we use a dimensionless form of Eq. (1). The rotation period of a small body is taken as the unit of time [T]. The equivalent radius of the central body is taken as the unit of length $[L]$. After introducing the parameters $[T]$ and $[L]$, the dimensionless form of orbital motion is expressed in Eq. (4).

$$
\left\{\begin{aligned}
\tilde{\boldsymbol{r}} & =\boldsymbol{r} /[L] \\
\tilde{\boldsymbol{\omega}} & =\boldsymbol{\omega} \cdot[T] \\
\hat{\boldsymbol{\omega}} & =\tilde{\boldsymbol{\omega}} /|\tilde{\boldsymbol{\omega}}|=\tilde{\boldsymbol{\omega}} / 2 \pi \\
\kappa & =G \sigma T_{\mathrm{A}}^{2} \\
\tilde{\boldsymbol{r}}^{\prime \prime} & +2 \tilde{\boldsymbol{\omega}} \times \tilde{\boldsymbol{r}}^{\prime}+\tilde{\boldsymbol{\omega}} \times(\tilde{\boldsymbol{\omega}} \times \tilde{\boldsymbol{r}}) \\
& =\tilde{\boldsymbol{r}}^{\prime \prime}+4 \pi \hat{\boldsymbol{\omega}} \times \tilde{\boldsymbol{r}}^{\prime}+4 \pi^{2} \hat{\boldsymbol{\omega}} \times(\hat{\boldsymbol{\omega}} \times \tilde{\boldsymbol{r}}) \\
& =\kappa\left(\sum_{e \in E S} L_{e} \boldsymbol{E}_{e} \cdot \tilde{\boldsymbol{r}}_{e}-\sum_{f \in F S} \theta_{f} \boldsymbol{F}_{f} \cdot \tilde{\boldsymbol{r}}_{f}\right)
\end{aligned}\right.
$$

Here, $\tilde{\boldsymbol{r}}$ represents the normalized position vector, $\tilde{\boldsymbol{\omega}}$ is the normalized angular velocity of the asteroid, and $\hat{\omega}$ is a unit vector. The dimensionless parameter $\kappa$ reflects the integrated influence of gravity and centrifugal force on the particle.

An index provided by the Laplacian of the polyhedron method is applied when detecting the particle's interaction with the asteroid surface. ${ }^{24)}$ The definition of the index $\Omega$ is

$$
\Omega=\sum_{f \in F S} \theta_{f}
$$

If the particle is outside the polyhedron, the index equals 0. If

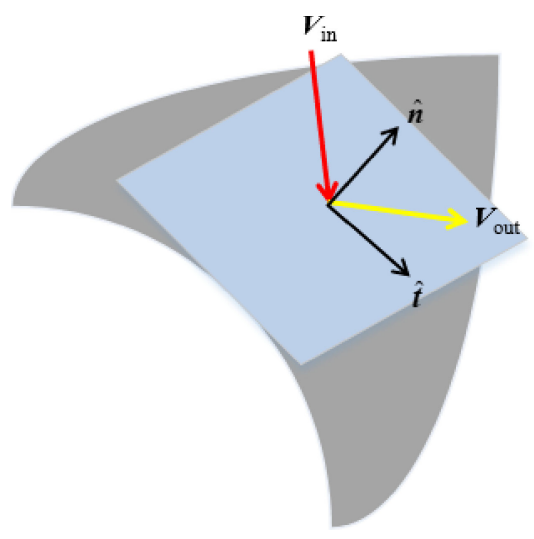

Fig. 2. Sketch map for velocity variation during a collision.

the particle is inside, $\Omega$ turns into $4 \pi$. When a particle interacts with the continuous surface, the collision is regarded as a fast, non-slip motion. The change of velocity depends on the kinematic restitution coefficients, which are applied to evaluate the outgoing velocity considering the energy dissipation. $^{25)}$

$$
\left\{\begin{array}{l}
\boldsymbol{V}_{\text {in }}=\boldsymbol{V}_{\text {nin }}+\boldsymbol{V}_{\text {tin }} \\
\boldsymbol{V}_{\text {out }}=-\varepsilon_{n} \boldsymbol{V}_{\text {nin }}+\varepsilon_{t} \boldsymbol{V}_{\text {tin }}
\end{array}\right.
$$

Here, $\boldsymbol{V}_{\text {in }}$ and $\boldsymbol{V}_{\text {out }}$ are the ingoing and outgoing velocities, respectively. $\boldsymbol{V}_{\text {nin }}$ and $\boldsymbol{V}_{\text {tin }}$ represent the normal and tangential components of $\boldsymbol{V}_{\text {in }}$. The variables $\varepsilon_{n}$ and $\varepsilon_{t}$ are the normal and tangential restitution coefficients, with their ranges satisfying

$$
\left\{\begin{array}{l}
0 \leq \varepsilon_{n} \leq 1 \\
-1 \leq \varepsilon_{t} \leq 1
\end{array}\right.
$$

Figure 2 illustrates the velocity variation of the collision. Through varying $\varepsilon_{n}$ and $\varepsilon_{t}$, different rebound velocities can be obtained. The collision model in Eqs. (6) and (7) will be adopted throughout the following discussions.

\subsection{Principle of the hopping trajectory design}

The trajectory design method assumes that the hopping rover starts moving from departure point $\mathrm{A}$ and plans to arrive at target point B after several hops. The crucial part of the method is to find the appropriate velocity increments that make the hopping rover eventually reach target point $\mathrm{B}$. The feasible velocity increments are computed using the shooting method, which takes the parabolic motion's initial velocity $\boldsymbol{V}_{0}$ as the initial value. Figure 3 is the corresponding schematic model of the parabolic motion.

In the parabolic motion, a particle starts to move from point A when time is zero, and it is assumed to arrive at target point B after $p$ times of hopping. The gravity $g$ is assumed along the negative $\boldsymbol{Z}$-axis, and its magnitude is constant. The unit vector from point A to point B is taken as the tangential direction $\boldsymbol{t}$. The vector $\boldsymbol{g}$ is projected in the tangential direction, and $\boldsymbol{g}_{n}$ is defined as the difference between $\boldsymbol{g}$ and $\boldsymbol{g}_{t}$. The unit vector of $\boldsymbol{g}_{n}$ is chosen to be the normal direction $\boldsymbol{n}$. Additionally, the hopping trajectories are all presumed to be in the same plane determined by $\boldsymbol{n}$ and $\boldsymbol{t}$.

According to the instantaneous collision model, the 


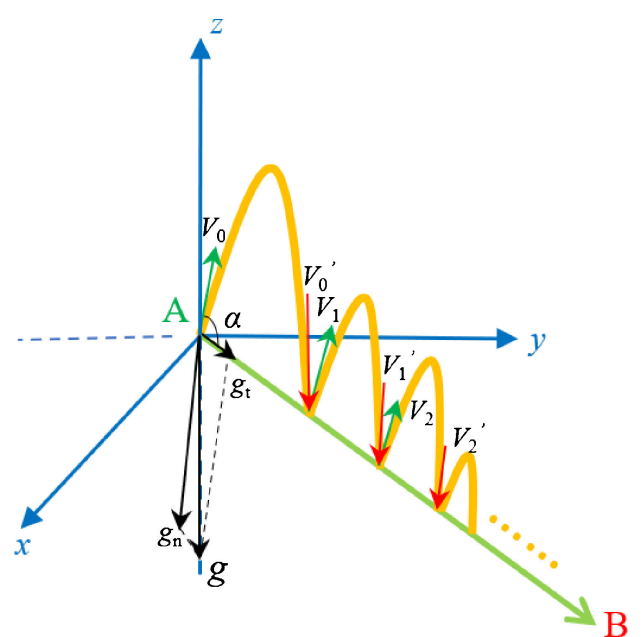

Fig. 3. The parabolic motion.

changes in velocity at the $k$ th rebounding point are

$$
\left\{\begin{array}{l}
\boldsymbol{V}_{(k) n}=\varepsilon_{n} \boldsymbol{V}_{(k-1) n^{\prime}} \\
\boldsymbol{V}_{(k) t}=\varepsilon_{t} \boldsymbol{V}_{(k-1) t^{\prime}}
\end{array} \quad(k=1,2, \ldots, p)\right.
$$

Here, $\boldsymbol{V}$ is the next hop's initial velocity, $\boldsymbol{V}^{\prime}$ represents the last hop's final velocity.

After the first hop, the magnitude of tangential velocity is

$$
\left\{\begin{array}{l}
V_{0 t}{ }^{\prime}=V_{0 t}+g_{t} t_{1} \\
t_{1}=\frac{2 V_{0 n}}{g_{n}}=\frac{2 V_{0 n}{ }^{\prime}}{g_{n}}
\end{array}\right.
$$

The first tangential hop distance $d_{1}$ is

$$
\begin{aligned}
d_{1} & =V_{0 t} t_{1}+\frac{1}{2} g_{t} t_{1}{ }^{2} \\
& =\frac{2 V_{0 t} V_{0 n}}{g_{n}}+\frac{2 g_{t} V_{0 n}{ }^{2}}{g_{n}{ }^{2}}
\end{aligned}
$$

Using Eqs. (8)-(10), the $k$ th hopping portion's time and distance can be deduced recursively. The value of the integer parameter $k$ is varied from 1 to $p$.

$$
\left\{\begin{aligned}
t_{k} & =\frac{2 \varepsilon_{n}{ }^{k-1} V_{0 n}}{g_{n}} \\
d_{k} & =\frac{2 \varepsilon_{n}{ }^{2(k-1)} V_{0 n} V_{0 t}}{g_{n}}+\frac{(4 k-2) V_{0 n}{ }^{2} g_{t} \varepsilon_{n}{ }^{2(k-1)}}{g_{n}{ }^{2}} \quad\left(\varepsilon_{n}=\varepsilon_{t}\right) \\
d_{k} & =\frac{2 \varepsilon_{n}{ }^{k-1} \varepsilon_{t}{ }^{k-1} V_{0 n} V_{0 t}}{g_{n}}+E+F \quad\left(\varepsilon_{n} \neq \varepsilon_{t}\right) \\
E & =\frac{2 V_{0 n}{ }^{2} g_{t} \varepsilon_{n}{ }^{2(k-1)}}{g_{n}{ }^{2}} \\
F & =\frac{4 V_{0 n}{ }^{2} g_{t}\left[\varepsilon_{n}{ }^{k-1} \varepsilon_{t}{ }^{k}-\varepsilon_{n}{ }^{2(k-1)} \varepsilon_{t}\right]}{g_{n}{ }^{2}\left(\varepsilon_{t}-\varepsilon_{n}\right)}
\end{aligned}\right.
$$

Here, $t_{k}$ and $d_{k}$ represent the time and tangential distance of the $k$ th hop, respectively. The letters $E$ and $F$ are the intermediate variables applied to make the expression more concise.

Based on $t_{k}$ and $d_{k}$, the cumulative transfer time $t_{\text {total }}$ and distance $d_{\text {total }}$ are

$$
\begin{aligned}
& \varepsilon_{n}=\varepsilon_{t}:\left\{\begin{aligned}
t_{\text {total }} & =\sum_{k=1}^{p} t_{k}=\frac{2 V_{0 n}\left(1-\varepsilon_{n}{ }^{p}\right)}{g_{n}\left(1-\varepsilon_{n}\right)} \\
d_{\text {total }} & =\sum_{k=1}^{p} d_{k}=\frac{2 V_{0 n} V_{0 t}}{g_{n}} \cdot \frac{1-\varepsilon_{n}{ }^{2 p}}{1-\varepsilon_{n}{ }^{2}}+L \\
L= & \frac{2 V_{0 n^{2} g_{t}}}{g_{n}{ }^{2}} \\
& {\left[\frac{1-(2 p-1) \varepsilon_{n}{ }^{2 p}}{1-\varepsilon_{n}{ }^{2}}+\frac{2 \varepsilon_{n}{ }^{2}\left(1-\varepsilon_{n}{ }^{2(p-1)}\right)}{\left(1-\varepsilon_{n}\right)^{2}}\right] }
\end{aligned}\right. \\
& \begin{array}{l}
t_{\text {total }}=\sum_{k=1}^{p} t_{k}=\frac{2 V_{0 n}\left(1-\varepsilon_{n}{ }^{p}\right)}{g_{n}\left(1-\varepsilon_{n}\right)} \\
d_{\text {total }}=\sum_{k=1}^{p} d_{k}
\end{array} \\
& =\frac{2 V_{0 n} V_{0 t}}{g_{n}} \cdot \frac{1-\varepsilon_{n}^{p} \varepsilon_{t}^{p}}{1-\varepsilon_{n} \varepsilon_{t}}+M+N-O \\
& M=\frac{2 V_{0 n}^{2} g_{t}}{g_{n}^{2}} \cdot \frac{1-\varepsilon_{n}^{2 p}}{1-\varepsilon_{n}^{2}} \\
& N=\frac{4 V_{0 n}{ }^{2} g_{t} \varepsilon_{t}\left(1-\varepsilon_{n}{ }^{p} \varepsilon_{t}{ }^{p}\right)}{g_{n}{ }^{2}\left(\varepsilon_{t}-\varepsilon_{n}\right)\left(1-\varepsilon_{n} \varepsilon_{t}\right)} \\
& O=\frac{4 V_{0 n}^{2} g_{t} \varepsilon_{t}\left(1-\varepsilon_{n}{ }^{2 p}\right)}{g_{n}{ }^{2}\left(\varepsilon_{t}-\varepsilon_{n}\right)\left(1-\varepsilon_{n}{ }^{2}\right)}
\end{aligned}
$$

Here, $L, M, N$, and $O$ are the intermediate variables.

Because the magnitudes of the restitution coefficients are between 0 and 1, Eqs. (12) and (13) can be simplified as

$\varepsilon_{n}=\varepsilon_{t}:\left\{\begin{array}{l}t_{\text {total }}=\frac{2 V_{0 n}}{g_{n}\left(1-\varepsilon_{n}\right)} \\ d_{\text {total }}=\frac{2 V_{0 n} V_{0 t}}{g_{n}} \cdot \frac{1}{1-\varepsilon_{n}{ }^{2}}+\frac{2 V_{0 n}{ }^{2} g_{t}}{g_{n}{ }^{2}} \cdot \frac{1+\varepsilon_{n}{ }^{2}}{\left(1-\varepsilon_{n}{ }^{2}\right)^{2}}\end{array}\right.$

$\varepsilon_{n} \neq \varepsilon_{t}:\left\{\begin{aligned} t_{\text {total }}= & \frac{2 V_{0 n}}{g_{n}\left(1-\varepsilon_{n}\right)} \\ d_{\text {total }}= & \frac{2 V_{0 n} V_{0 t}}{g_{n}} \cdot \frac{1}{1-\varepsilon_{n} \varepsilon_{t}} \\ & +\frac{2 V_{0 n}{ }^{2} g_{t}}{g_{n}{ }^{2}} \cdot \frac{1+\varepsilon_{t} \varepsilon_{n}}{\left(1-\varepsilon_{n}{ }^{2}\right)\left(1-\varepsilon_{n} \varepsilon_{t}\right)}\end{aligned}\right.$

After simplification, Eqs. (14) and (15) of $\varepsilon_{n}=\varepsilon_{t}$ and $\varepsilon_{n} \neq \varepsilon_{t}$ can be unified as

$$
\left\{\begin{aligned}
t_{\text {total }} & =\frac{2 V_{0 n}}{g_{n}\left(1-\varepsilon_{n}\right)} \\
d_{\text {total }} & =\frac{2 V_{0 n} V_{0 t}}{g_{n}\left(1-\varepsilon_{n} \varepsilon_{t}\right)}+\frac{2 V_{0 n}{ }^{2} g_{t}\left(1+\varepsilon_{t} \varepsilon_{n}\right)}{g_{n}{ }^{2}\left(1-\varepsilon_{n}{ }^{2}\right)\left(1-\varepsilon_{n} \varepsilon_{t}\right)}
\end{aligned}\right.
$$

Based on the angle $\alpha$ between $V_{0}$ and $\boldsymbol{t}$, the expression of $d_{\text {total }}$ in Eq. (16) can be rewritten as 


$$
\left\{\begin{aligned}
V_{0 n} & =V_{0} \sin \alpha \\
V_{0 t} & =V_{0} \cos \alpha \\
d_{\text {total }} & =\frac{2 V_{0 n} V_{0 t}}{g_{n}\left(1-\varepsilon_{n} \varepsilon_{t}\right)}+\frac{2 V_{0 n}{ }^{2} g_{t}\left(1+\varepsilon_{t} \varepsilon_{n}\right)}{g_{n}{ }^{2}\left(1-\varepsilon_{n}{ }^{2}\right)\left(1-\varepsilon_{n} \varepsilon_{t}\right)} \\
& =\frac{\sin 2 \alpha g_{n}\left(1-\varepsilon_{n}{ }^{2}\right)+g_{t}\left(1+\varepsilon_{n} \varepsilon_{t}\right)(1-\cos 2 \alpha)}{g_{n}{ }^{2}\left(1-\varepsilon_{n}{ }^{2}\right)\left(1-\varepsilon_{n} \varepsilon_{t}\right)} V_{0}^{2}
\end{aligned}\right.
$$

If knowing the departure point $\mathrm{A}$ and target point $\mathrm{B}$, the direction of $\boldsymbol{n}$ and $\boldsymbol{t}$, gravity at the initial point, and distance $d_{\text {total }}$ can be determined. Therefore, the initial velocity of the parabolic motion becomes the only unknown variable in Eq. (17), which can be expressed inversely from the equation of $d_{\text {total }}$

$$
\left\{\begin{aligned}
V_{0} & =\sqrt{\frac{d_{\text {total }} g_{n}{ }^{2}\left(1-\varepsilon_{n}{ }^{2}\right)\left(1-\varepsilon_{n} \varepsilon_{t}\right)}{\sin 2 \alpha g_{n}\left(1-\varepsilon_{n}{ }^{2}\right)+\left(1+\varepsilon_{n} \varepsilon_{t}\right) g_{t}(1-\cos 2 \alpha)}} \\
\boldsymbol{V}_{0} & =V_{0} \sin \alpha \boldsymbol{n}+V_{0} \cos \alpha \boldsymbol{t}
\end{aligned}\right.
$$

If the calculated $V_{0}$ in Eq. (18) is less than the escape velocity, the parabolic motion's initial velocity can be taken as the initial iteration value of the shooting method. However, in some simulations, the particle cannot be transferred to the target point by applying only one velocity increment. Therefore, numerous iterations of the shooting method are needed. In the first iteration, the departure point is set to be the given initial point A. Afterward, the previous hopping trajectory terminal is set as the next iteration's departure point. If position error is acceptable, the iteration ends and the final trajectory is obtained. In this study, the normalized threshold of position error is set to be $10^{-4}$ (about $0.19 \mathrm{~m}$ ), and this threshold can be reduced by improving the precision of the polyhedron model.

The pseudo-code of the planning method is listed in Table 1. After several iterations of the shooting method, the hopping rover eventually arrives to the target point. Additionally, the design method only retrieves the shortest feasible hopping trajectories off the surface. Thus, there is no need to check whether or not the solutions are unreasonable, such as the trajectory penetrating the surface or the non-shortest path.

\subsection{Construction of the asteroid terrain}

Construction of the asteroid polyhedron model involves rock generation, point cloud simplification, ${ }^{26)}$ and Delaunay triangulation.

Cube, octahedron, dodecahedron, and icosahedron are applied and deformed randomly to generate the rocks. ${ }^{27)}$ The rocks' centroids are transferred to the surface when merging them with the original polyhedron model. Afterward, the tetrahedron detection method is applied to delete the rock vertices beneath the surface. The detection process is as follows: Lots of triangular facets construct the polyhedron model. One triangular facet and the centroid of the polyhedron model can form a tetrahedron. It is assumed that the coordinates of the tetrahedral vertices are
Table 1. Process of the hopping trajectory planning.

Input: $\boldsymbol{r}_{\mathrm{A}}, \boldsymbol{r}_{\mathrm{B}}, \boldsymbol{g} \in \mathrm{R}^{3}$, position error $s$ and its threshold $s_{\mathrm{h}}$

Output: the solved actual initial velocity increments and the final position deviation.

Do while $\left(s>s_{\mathrm{h}}\right)$

1: Calculate the velocity $\boldsymbol{V}_{0}$ at the departure point from the parabolic motion, which is taken to be the initial value of the shooting method.

2: Use the shooting method to solve the actual initial velocity.

2.1 Compute the Jacobian matrix and put $\boldsymbol{V}_{0}$ in the first iteration.

2.2 Determine the increment direction, and add it to $\boldsymbol{V}_{0}$, update the step bound.

2.3 Test for successful iteration, update the initial velocity and the position deviation $\Delta \boldsymbol{x}$ if successful; if not, do not update.

2.4 Test for termination, if the shooting method comes to the terminal, go to Step 3. Otherwise, continue to Step 2.5.

2.5 Determine if recalculation of the Jacobian matrix is necessary, return to Step 2.1 if necessary while calculating the rank-one modification to the Jacobian matrix, and return to Step 2.2 if unnecessary.

3: One iteration of the shooting method ends. Record the initial velocity increment. Update the departure point and the position error $s$. End do

$$
\begin{array}{ll}
\boldsymbol{v}_{1}=\left[x_{1}, y_{1}, z_{1}\right]^{\mathrm{T}}, & \boldsymbol{v}_{2}=\left[x_{2}, y_{2}, z_{2}\right]^{\mathrm{T}} \\
\boldsymbol{v}_{3}=\left[x_{3}, y_{3}, z_{3}\right]^{\mathrm{T}}, & \boldsymbol{v}_{4}=[0,0,0]^{\mathrm{T}}
\end{array}
$$

Here $\boldsymbol{v}_{1}, \boldsymbol{v}_{2}$, and $\boldsymbol{v}_{3}$ represent the three vertices of the triangular facet, and $\boldsymbol{v}_{4}$ is the centroid.

When a rock vertex $\boldsymbol{v}_{0}=[x, y, z]^{\mathrm{T}}$ is inside the tetrahedron, determinants formed by the coordinates of $\boldsymbol{v}_{0}, \boldsymbol{v}_{1}, \boldsymbol{v}_{2}$, $\boldsymbol{v}_{3}$, and $\boldsymbol{v}_{4}$ will have the same sign

$$
\begin{aligned}
& D_{1}=\left|\begin{array}{llll}
x_{1} & y_{1} & z_{1} & 1 \\
x_{2} & y_{2} & z_{2} & 1 \\
x_{3} & y_{3} & z_{3} & 1 \\
0 & 0 & 0 & 1
\end{array}\right|, D_{2}=\left|\begin{array}{cccc}
x & y & z & 1 \\
x_{2} & y_{2} & z_{2} & 1 \\
x_{3} & y_{3} & z_{3} & 1 \\
0 & 0 & 0 & 1
\end{array}\right| \\
& D_{3}=\left|\begin{array}{cccc}
x_{1} & y_{1} & z_{1} & 1 \\
x & y & z & 1 \\
x_{3} & y_{3} & z_{3} & 1 \\
0 & 0 & 0 & 1
\end{array}\right|, D_{4}=\left|\begin{array}{cccc}
x_{1} & y_{1} & z_{1} & 1 \\
x_{2} & y_{2} & z_{2} & 1 \\
x & y & z & 1 \\
0 & 0 & 0 & 1
\end{array}\right| \\
& D_{5}=\left|\begin{array}{llll}
x_{1} & y_{1} & z_{1} & 1 \\
x_{2} & y_{2} & z_{2} & 1 \\
x_{3} & y_{3} & z_{3} & 1 \\
x & y & z & 1
\end{array}\right|
\end{aligned}
$$

Here, $D_{1}-D_{5}$ represent the determinants composed of $\boldsymbol{v}_{0}, \boldsymbol{v}_{1}$, $\boldsymbol{v}_{2}, \boldsymbol{v}_{3}$, and $\boldsymbol{v}_{4}$, respectively. By computing the five determinants, the rocky vertices below the asteroid surface can be determined and deleted.

After deleting the impropriate rock vertices, a new integrated point cloud is obtained. Afterward, the new point cloud is simplified and triangulated to generate the rocky polyhedron model. The merged point clouds and triangularization result are shown in Fig. 4. 


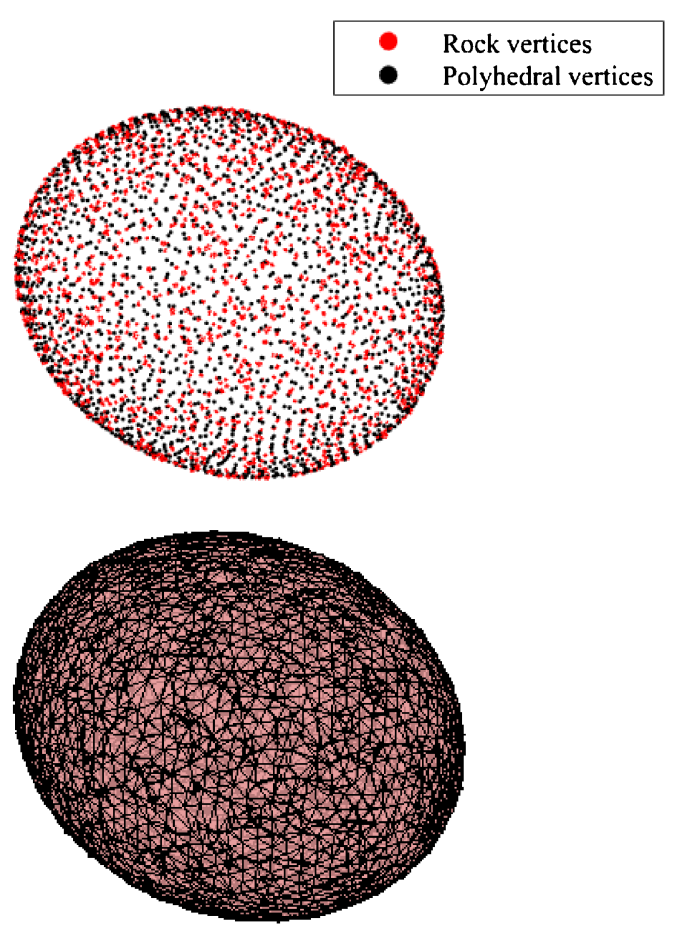

Fig. 4. Integrated point cloud and the triangulation process.

\section{Numerical Simulations}

\subsection{Applications to the smooth polyhedron model}

In this study, comet 133P/Elst-Pizarro is applied as the central body's background. Because existing missions have not explored comet 133P, a detailed model of this comet is not currently available. Therefore, a homogeneous ellipsoid is applied to simplify $133 \mathrm{P}$ based on the comet's observed rough statistics, including rotation period, density, and size parameters. The proposed design method is first applied to the smooth polyhedron model of the ellipsoid without rocks to explain and show the preliminary planned hopping trajectories. The collision restitution coefficients are set to be 0.5 in all simulations.

Comet 133P has an effective radius $R_{\mathrm{e}}$ of $1.9 \mathrm{~km}$ with estimated semiaxes of $\mathrm{a}=2.3 \mathrm{~km}$ and $\mathrm{b}=1.6 \mathrm{~km} .{ }^{28)}$ When taking an ellipsoid model to simulate 133P, two semiaxes of the ellipsoid are $2.3 \mathrm{~km}$ and $1.6 \mathrm{~km}$, respectively. Based on the identical volume calculated using the equivalent sphere and estimated ellipsoid, another semiaxis of the ellipsoid is obtained from Eq. (21)

$$
\frac{4 \pi}{3} \mathrm{abc}=\frac{4 \pi}{3} R_{\mathrm{e}}^{3}
$$

Here, c represents the ellipsoid's third semiaxis, the value of which is calculated to be $1.86 \mathrm{~km}$ based on the known semiaxes a and $\mathrm{b}$.

Two sets of the initial point A and target point B are chosen from the smooth ellipsoid surfaces. In Scenario I, the initial point and target point are set as $r_{\mathrm{A} 1}=[0.91,0.17,1.46]^{\mathrm{T}} \mathrm{km}$ and $r_{\mathrm{B} 1}=[-0.30,0.32,1.56]^{\mathrm{T}} \mathrm{km}$, respectively. The latitudes of the two points are similar, while their longitudes are different. In Scenario II, the initial point and target point are chosen to be $r_{\mathrm{A} 2}=[0.095,0.91,1.40]^{\mathrm{T}} \mathrm{km}$ and $r_{\mathrm{B} 2}=$ $[0.095,1.77,0.49]^{\mathrm{T}} \mathrm{km}$, respectively. The two points have similar longitudes and different latitudes. Additionally, the Euclidean distances between the initial points and target points are approximately $1.23 \mathrm{~km}$ in the two cases.

Figure 5(a) shows the designed trajectory in Scenario I, where A1 and B1 represent the initial point and target point, respectively. Figure 6(a) illustrates the corresponding enlarged trajectory. The arrow represents the applied velocity increment, indicating that one iteration of the shooting method begins and the subsequent hopping trajectories before the next arrow belong to the free rebounding. The number of arrows reflects the number of hops with control. Similarly, Fig. 5(b) illustrates the trajectory from initial point A2 to target point B2 in Scenario II.

\subsection{Applications to the rocky polyhedron model}

After simplifying and triangulating the integrated point cloud, a rocky polyhedron model closer to the rough surfaces than the original smooth model is obtained. Similar to the smooth model simulations, two hopping trajectories are designed under the rocks' effect in Scenario III and Scenario IV, respectively. Two sets of the surface initial points and target points are chosen near the corresponding points of the smooth model: $r_{\mathrm{A} 3}=[0.91,0.17,1.44]^{\mathrm{T}} \mathrm{km}$ and $r_{\mathrm{B} 3}=$ $[-0.30,0.32,1.56]^{\mathrm{T}} \mathrm{km}$, and $r_{\mathrm{A} 4}=[0.095,0.89,1.39]^{\mathrm{T}} \mathrm{km}$ and $r_{\mathrm{B} 4}=[0.11,1.77,0.50]^{\mathrm{T}} \mathrm{km}$. The distance between the initial and target point equals $1.23 \mathrm{~km}$, which is identical to that of the smooth model.

In Scenario III, the designed trajectory and its detailed velocity increment analysis are shown in Fig. 5(c) and Fig. 6(b), respectively. Afterward, Fig. 5(d) exhibits the planned trajectory from A4 to B4.

\subsection{Discussion and comparison}

A parametric analysis of the designed trajectories in the four scenarios was conducted to compare the design processes. The magnitudes of the applied velocity increments and the corresponding times in the two smooth scenarios were recorded and are shown in Fig. 7(a). Some critical parameters are chosen to describe the planned trajectories' characteristics, including the sum of the velocity increments' magnitudes, total transfer time, number of controlled hops, and final position error. Among them, the sum of the velocity increments' magnitudes reflects the designed trajectory's energy consumption. The transfer time describes the time required for the hopping rover to move from the initial point to the target area. The number of controlled hops indicates the number of applied velocity increments, reflecting the operational complexity of the designed trajectory. The final position error between the trajectory terminal and target point represents the hopping trajectory's accuracy. The values of these parameters in Scenario I and Scenario II are listed in Table 2. Similarly, the velocity increments applied in the two rocky scenarios are analyzed in Fig. 7(b). The properties of the planned trajectories in Scenario III and Scenario IV are listed in Table 3, with the same parameters as those in Table 2.

In Table 2 and Table 3, the final position errors are all less 


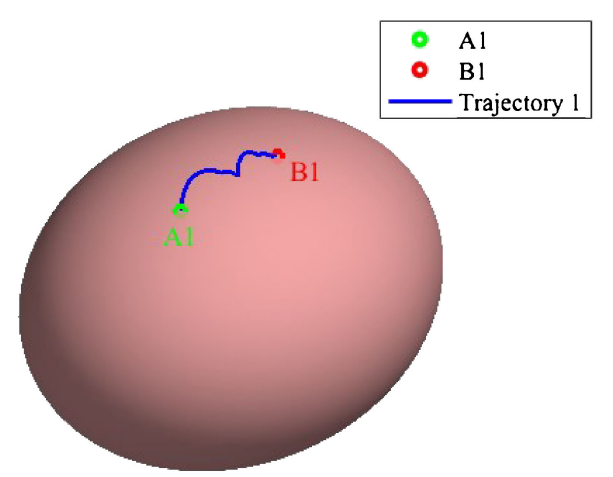

(a) Designed hopping trajectory in Scenario I.

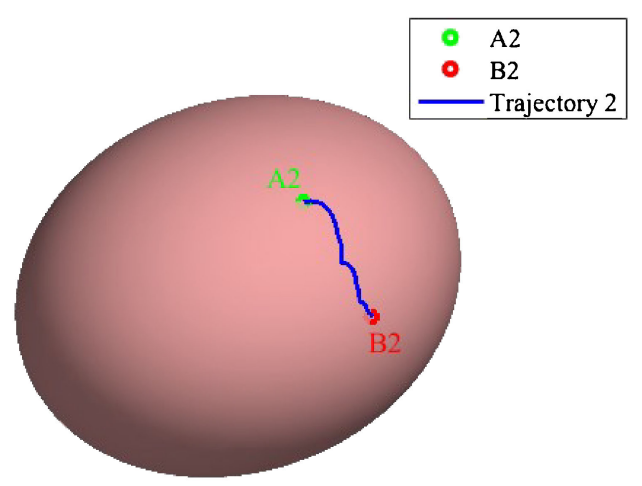

(b) Designed hopping trajectory in Scenario II.

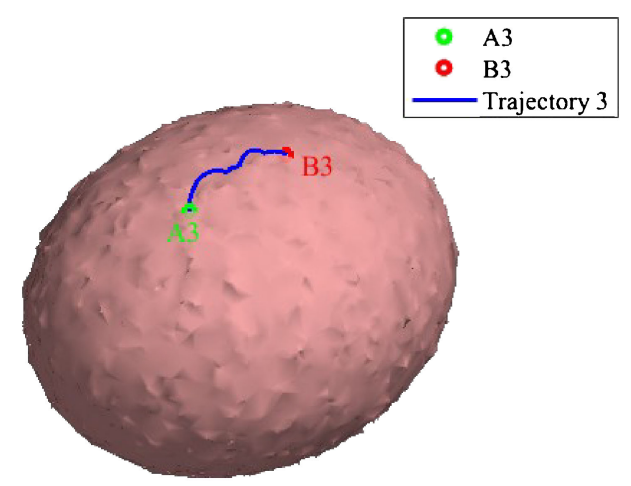

(c) Designed hopping trajectory in Scenario III.

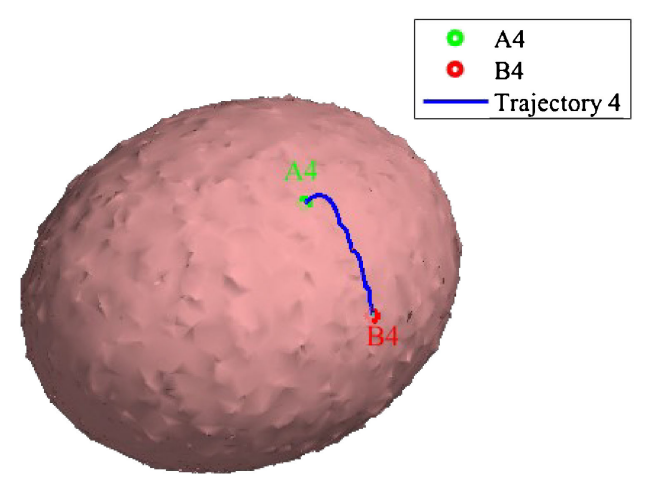

(d) Designed hopping trajectory in Scenario IV.

Fig. 5. Designed trajectories for the four different scenarios.

than $20 \mathrm{~cm}$, reflecting the proposed method's effectiveness. Comparing the two model's designed trajectories in Fig. 7, their velocity increment variation trends are similar. However, the sum of the velocity increments' magnitudes increases from $1.59 \mathrm{~m} / \mathrm{s}$ in Scenario I (smooth) to $1.81 \mathrm{~m} / \mathrm{s}$

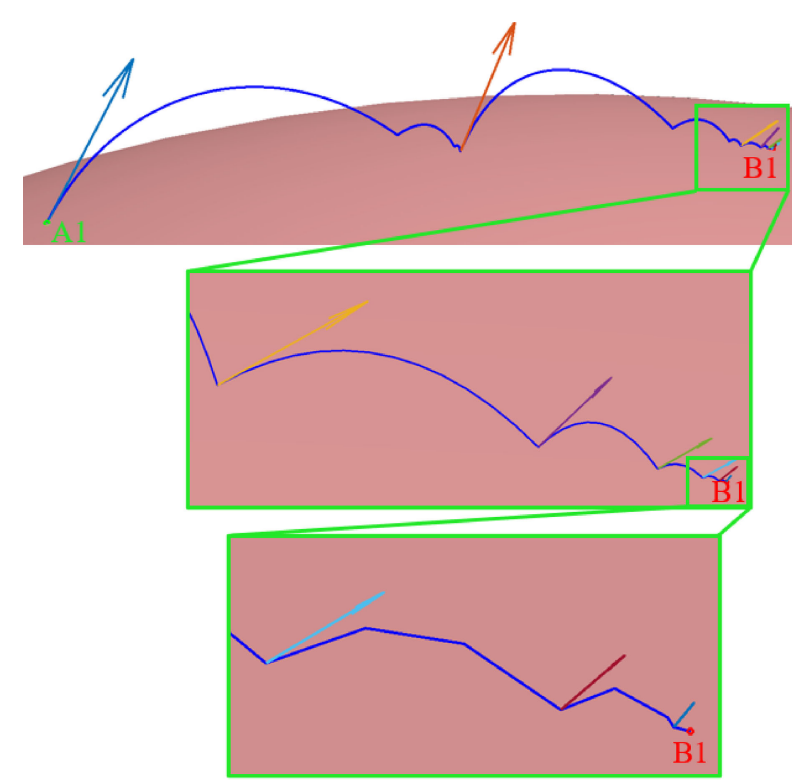

(a) An enlarged illustration of the trajectory in Scenario I.

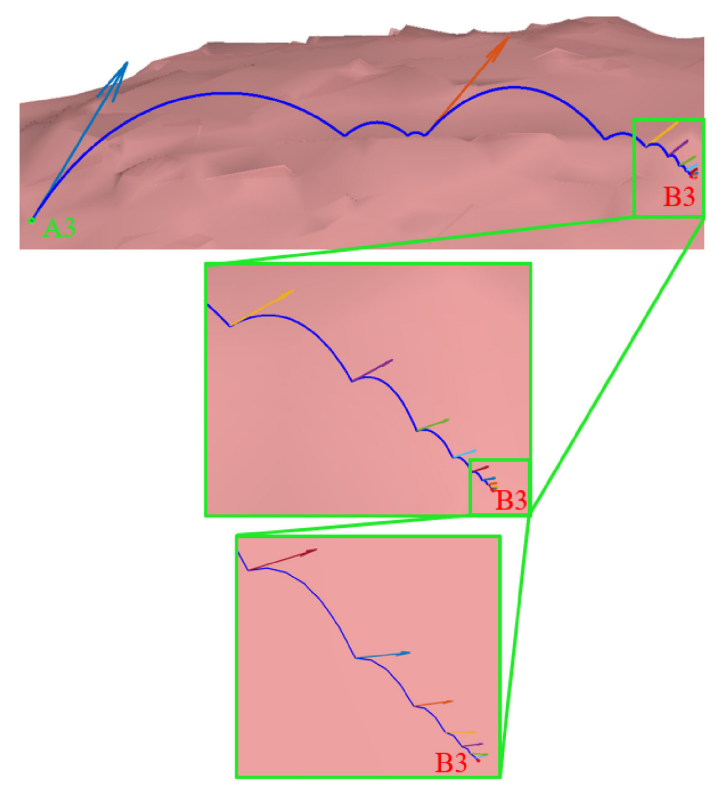

(b) An enlarged illustration of the trajectory in Scenario III.

Fig. 6. Enlarged illustrations of the trajectories in Scenario I and Scenario III

in Scenario III (rocky), and changes from $1.04 \mathrm{~m} / \mathrm{s}$ in Scenario II (smooth) to $1.07 \mathrm{~m} / \mathrm{s}$ in Scenario IV (rocky). The changes indicate that the sum of the velocity increments' magnitudes becomes larger as a result of being influenced by surface rock. Simultaneously, the number of controlled hops in the first set of surface points increased from 8 to 13 and decreased from 13 to 10 in the second set of points when considering the terrain roughness.

This contrastive analysis shows that the influence of rocks on trajectory design is evident while not being unilateral, which means that the effect of terrain roughness on trajectory evolution is random and uncertain. For example, different shaped rocks may impede the particle in advance and increase the number of controlled hops required. In contrast, the rocks may change the particle's original velocity direc- 


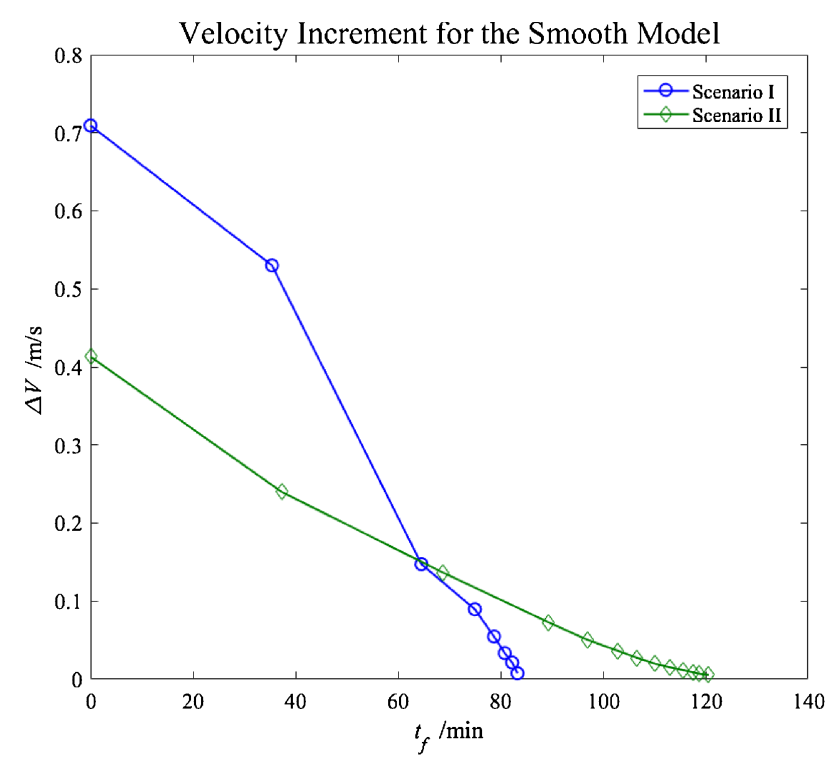

(a) Velocity increment in Scenario I and Scenario II.

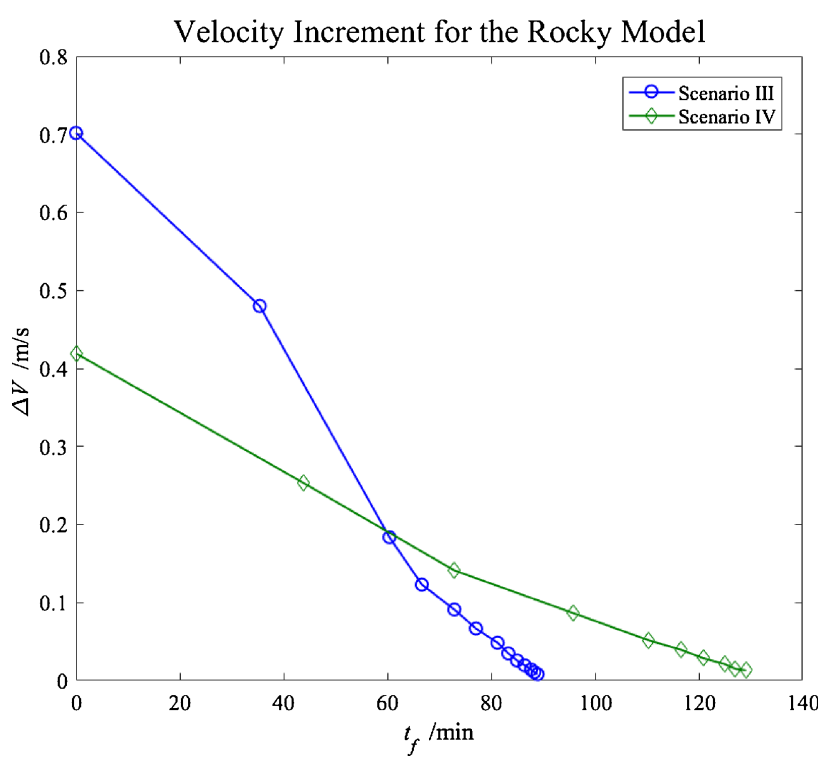

(b) Velocity increment in Scenario III and Scenario IV.

Fig. 7. Velocity increment in the four different scenarios.

tion at the time of collision and help it move towards the target area accidentally, which may lead to a decrease in the number of hops required. It is more complicated to analyze the sum of the velocity increments' magnitudes than the number of controlled hops. Since rocks influence particle motion randomly, the individual hindrance or promotion effect should be considered accumulatively to obtain the change in the sum of velocity magnitudes.

In the four scenarios, the initial and target points have similar latitudes in Scenario I and Scenario III. In contrast, the longitudes of the two points are similar in Scenario II and Scenario IV. The sums of the velocity increments' magnitudes in Scenario I and Scenario III are larger than those of Scenario II and Scenario IV. This phenomenon demonstrates that the position relationship between the initial and target points also influences the designed velocity increments. To further explain the influence, the particle's free locomotion
Table 2. Trajectory parameters for the smooth model.

\begin{tabular}{lcc}
\hline Parameter & Scenario I & Scenario II \\
\hline Sum of velocity increment & $1.59 \mathrm{~m} / \mathrm{s}$ & $1.04 \mathrm{~m} / \mathrm{s}$ \\
$\quad$ magnitudes & $83.72 \mathrm{~min}$ & $121.24 \mathrm{~min}$ \\
Total transfer time & 8 & 13 \\
Number of controlled hops & $0.12 \mathrm{~m}$ & $0.18 \mathrm{~m}$ \\
Final position error & & \\
\hline
\end{tabular}

Table 3. Trajectory parameters for the rocky model.

\begin{tabular}{ccc}
\hline Parameter & Scenario III & Scenario IV \\
\hline Sum of velocity increment & $1.81 \mathrm{~m} / \mathrm{s}$ & $1.07 \mathrm{~m} / \mathrm{s}$ \\
magnitudes & $83.34 \mathrm{~min}$ & $130.10 \mathrm{~min}$ \\
Total transfer time & 13 & 10 \\
Number of controlled hops & $0.17 \mathrm{~m}$ & $0.18 \mathrm{~m}$ \\
Final position error & & \\
\hline
\end{tabular}

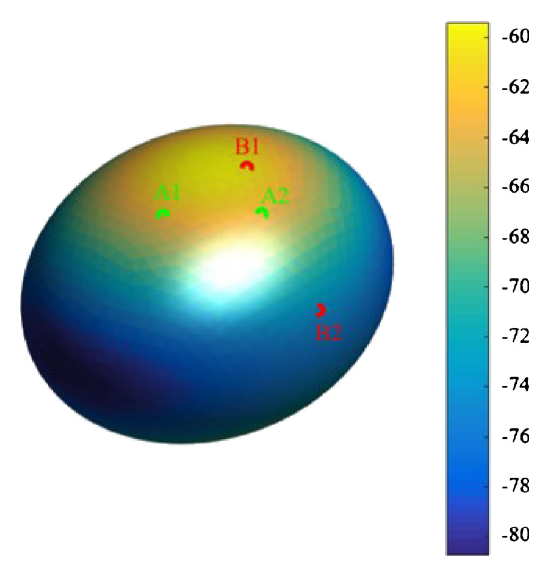

Fig. 8. The effective potential of the smooth model.

property reported by Zhang et al. ${ }^{29)}$ is applied. This reference declares that particles moved freely towards the low effective potential area of the asteroid Bennu regardless of the initial position. Suppose the lander is free in an area with high effective potential (i.e., high latitude areas on Bennu). In such a case, the lander may be easy to move a long distance towards a low effective potential portion (i.e., area of Bennu near the equator).

Based on a particle's free motion regularity, it can be assumed that the influence of position relationship on trajectory design is, in essence, caused by the changes in effective potential. In order to verify this hypothesis, the global normalized effective potentials are illustrated in Figs. 8 and 9, corresponding to the smooth model and rocky model, respectively. The applied initial points and target points are also plotted in the two figures.

Taking the smooth model as an example, the normalized effective potentials of $\mathrm{A} 1$ and $\mathrm{B} 1$ are -63.49 and -60.84 , respectively, which are almost the same. However, the normalized effective potentials of $\mathrm{A} 2$ and $\mathrm{B} 2$ are -63.76 and -74.88 , respectively, which means that the target point has a lower effective potential in this case. Therefore, the particle itself has the free-motion characteristic of hopping towards 


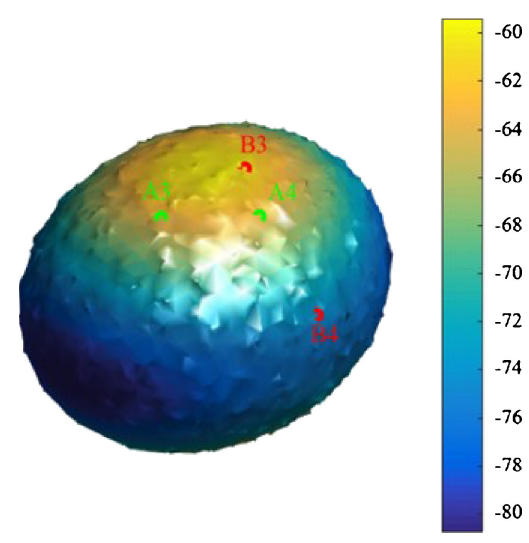

Fig. 9. The effective potential of the rocky model.

the target point area in Scenario II, which leads to the smaller sum of velocity increment magnitudes. This influence can be generalized to all of the trajectory design cases over different asteroids: If the hopping rover transfers from a point with the high effective potential to a low effective potential area, the sum of the needed velocity increments' magnitudes is relatively smaller.

\section{Conclusion}

This paper presents a hopping trajectory planning method over smooth and rocky polyhedron model surfaces. The core algorithm in the design method constantly treats the terminal of the last hop as the departure point of the next hop and applies the shooting method to search the proper velocity increment until the final position error is acceptable. The initial value of the shooting method is obtained based on simple parabolic motion.

By taking an ellipsoid to simplify comet 133P, smooth and rocky ellipsoidal polyhedron models are constructed. Afterward, four different trajectories are planned without and with the influence of rocks. These trajectories' final position errors are all less than $20 \mathrm{~cm}$, indicating the design method's effectiveness. Through comparing the four trajectories, it is found that the addition of rocks increases the randomness of the trajectory evolution. Additionally, a particle's free characteristics caused by the change in effective potential also influences the sum of the needed velocity increment magnitudes.

The discussions in this paper may provide some references for future missions that utilize hopping rovers. For example, a detailed preliminary characterization of an asteroid's terrain is an essential prerequisite for precise trajectory design. When it is necessary to reduce energy consumption, a target point for which the effective potential is lower than that of the initial point should be given priority. Future studies will discuss the coupling motion taking into consideration the change in hopping rover attitude to make up for drawbacks in the particle model. The interactions between differentshaped hopping rovers and asteroid surfaces will also be analyzed.

\section{Acknowledgments}

This work was supported by the National Natural Science Foundation of China (No. 11972075) and the Open Research Fund of Key Laboratory of Space Utilization and the Strategic Priority Research Program on Space Science, Chinese Academy of Sciences (No. LSU-KFJJ-2018-01 and XDA 15020300).

\section{References}

1) Bibring, J.-P., Langevin, Y., and Carter, J.: 67P/Churymov-Gerasimenko Surface Properties as Derived from CIVA Panoramic Images, Science, 349, 6247 (2015), aab0671.

2) Boehnhardt, H., Bibring, J.-P., and Apathy, I.: The Philae Lander Mission and Science Overview, Philos. Trans. R. Soc. A: Math. Phys. Eng. Sci., 375, 2097 (2017), 20160248.

3) Ulamec, S., Kucherenko, V., and Biele, J.: Hopper Concepts for Small Body Landers, Adv. Space Res., 47, 3 (2011), pp. 428-439.

4) Spohn, T., Knollenberg, J., Ball, A. J., and Banaszkiewicz, M.: Thermal and Mechanical Properties of the Near-surface Layers of Comet 67P/C-G, Science, 349, 6247 (2015), aab0464.

5) Biele, J., Ulamec, S., and Maibaum, M.: Cometary Science. The Landing(s) of Philae and Inference about Comet Surface Mechanical Properties, Science, 349, 6247 (2015), aaa9816.

6) Bibring, J.-P., Rosenbauer, H., and Boehnhardt, H.: The Rosetta Lander ("Philae") Investigations, Space Sci. Rev., 128 (2007), pp. 205-220.

7) Yoshikawa, K., Sawada, H., and Kikuchi, S.: Modeling and Analysis of Hayabusa2 Touchdown, Astrodynamics, 4, 2 (2020), pp. 119-135.

8) Ogawa, N., Terui, F., and Mimasu, Y.: Image-based Autonomous Navigation of Hayabusa2 Using Artificial Landmarks: The Design and Brief In-flight Results of the First Landing on Asteroid Ryugu, Astrodynamics, 4, 2 (2020), pp. 89-103.

9) Tsuda, Y., Saiki, T., and Terui, F.: Hayabusa2 Mission Status: Landing, Roving and Cratering on Asteroid Ryugu, Acta Astronautica, 171 (2020), pp. 42-54.

10) Zeng, X., Wen, T., Yu, Y., and Circi, C.: Potential Hop Reachable Domain over Surfaces of Small Bodies, Aerospace Science Technology, 112 (2021), 106600

11) Bellerose, J. and Scheeres, D. J.: Dynamics and Control for Surface Exploration of Small Bodies, AIAA/AAS Astrodynamics Specialist Conference and Exhibit, Honolulu, Hawaii, AIAA 2008-6251, 2008.

12) Liu, Y., Zhu, S., and Cui, P.: Hopping Trajectory Optimization for Surface Exploration on Small Bodies, Adv. Space Res., 60, 1 (2017), pp. 90-102.

13) Hockman, B. and Pavone, M.: Stochastic Motion Planning for Hopping Rovers on Small Solar System Bodies, Robotics Research, Amato, N., Hager, G., Thomas, S., and Torres-Torriti, M. (ed.), Springer International Publ, Cham, Switzerland, 2020, pp. 877-893.

14) Kalita, H. and Thangavelautham, J.: Motion Planning on an Asteroid Surface with Irregular Gravity Fields, Adv. Astronaut. Sci. Guid. Navigation Control, 169 (2019), pp. 69-79.

15) Mahaney, W. and Kapran, B.: Landforms on Asteroid 25143-Itokawa: A Geomorphological Perspective on Periglacial Origin and Meltwater History, Geomorphology, 108, 3-4 (2009), pp. 321-323.

16) Lauretta, D. S., DellaGiustina, D. N., Bennett, C. A., and Golish, D. R.: The Unexpected Surface of Asteroid (101955) Bennu, Nature, 568, 7750 (2019), pp. 55-60.

17) Michikami, T., Honda, C., and Miyamoto, H.: Boulder Size and Shape Distributions on Asteroid Ryugu, Icarus, 331 (2019), pp. 179-191.

18) Van Wal, S., Tsuda, Y., Yoshikawa, K., Miura, A., Tanaka, S., and Scheeres, D.: Prearrival Deployment Analysis of Rovers on Hayabusa2 Asteroid Explorer, J. Spacecr. Rockets, 55, 4 (2018), pp. 797-817.

19) Yu, Y. and Baoyin, H.: Modeling of Migrating Grains on Asteroid's Surface, Astrophys. Space Sci., 355, 1 (2015), pp. 43-56.

20) Yoshimitsu, T. and Kubota, T.: "Hayabusa 2" MINERVA-II Rover's Robot Technology and Asteroid Surface Exploration $(<$ Special Feature $>$ Further Challenge, Asteroid Probe Hayabusa 2), J. Mech. Soc. Jpn., 122, 1213 (2019), pp. 26-29 (in Japanese). 
Trans. Japan Soc. Aero. Space Sci., Vol. 64, No. 4, 2021

21) Wen, T., Zeng, X., Circi, C., and Gao, Y.: Hop Reachable Domain on Irregularly Shaped Asteroids, J. Guid. Control Dynam., 43, 7 (2020), pp. 1269-1283.

22) Yu, Y. and Baoyin, H.: Routing the Asteroid Surface Vehicle with Detailed Mechanics, Acta Mechanica Sinica, 30, 3 (2014), pp. 301-309.

23) Werner, R.: The Gravitational Potential of a Homogeneous Polyhedron or Don't Cut Corners, Celest. Mech. Dynam. Astron., 59, 3 (1994), pp. 253-278.

24) Werner, R. and Scheeres, D. J.: Exterior Gravitation of a Polyhedron Derived and Compared with Harmonic and Mascon Gravitation Representations of Asteroid 4769 Castalia, Celest. Mech. Dynam. Astron., 65, 3 (1996), pp. 313-344.

25) Lorda, L., Canalias, E., Martin, T., and Biele, J.: Analyses of the Descent and Bouncing Trajectories to Support the Landing Site Selection, 26th International Symposium on Space Flight Dynamics, Matsuyama,
Japan, ISSFD-2017-032, 2017

26) Han, H., Han, X., Sun, F., and Huang, C.: Point Cloud Simplification with Preserved Edge Based on Normal Vector, Optik, 126, 19 (2015), pp. 2157-2162.

27) Tardivel, S., Scheeres, D. J., and Michel, P.: Contact Motion on Surface of Asteroid, J. Spacecr. Rockets, 51, 6 (2014), pp. 1857-1871.

28) Hsieh, H., Jewitt, D., and Yanga, R.: Albedos of Main-Belt Comets 133P/Elst-Pizarro and 176P/LINEAR, Astrophys. J., 694, 2 (2009), pp. L111-L114.

29) Zhang, Y., Zeng, X., and Circi, C.: The Motion of Surface Particles for the Asteroid 101955 Bennu, Acta Astronautica, 163 (2019), pp. 3-10.

Matteo Ceriotti Associate Editor 